\title{
Biochemical Machines for the Interconversion of Mutual Information and Work
}

\author{
Thomas McGrath, ${ }^{1}$ Nick S. Jones, ${ }^{1}$ Pieter Rein ten Wolde, ${ }^{2}$ and Thomas E. Ouldridge ${ }^{3 *}$ \\ ${ }^{1}$ Department of Mathematics, Imperial College London, London SW7 2AZ, United Kingdom \\ ${ }^{2}$ FOM Institute AMOLF, Science Park 104, 1098 XE Amsterdam, Netherlands \\ ${ }^{3}$ Department of Bioengineering, Imperial College London, London SW7 2AZ, United Kingdom \\ (Received 18 April 2016; revised manuscript received 20 September 2016; \\ published 10 January 2017; publisher error corrected 12 January 2017)
}

\begin{abstract}
We propose a physically realizable information-driven device consisting of an enzyme in a chemical bath, interacting with pairs of molecules prepared in correlated states. These correlations persist without direct interaction and thus store free energy equal to the mutual information. The enzyme can harness this free energy, and that stored in the individual molecular states, to do chemical work. Alternatively, the enzyme can use the chemical driving to create mutual information. A modified system can function without external intervention, approaching biological systems more closely.
\end{abstract}

DOI: 10.1103/PhysRevLett.118.028101

Organisms exploit correlations in their environment to survive and grow. This fact holds across scales, from bacterial chemotaxis, which leverages the spatial clustering of food molecules [1,2], to the loss of leaves by deciduous trees, which is worthwhile because sunlight exposure is highly correlated from day to day. Evolution itself relies on correlations across time and space; otherwise, a mutation which is beneficial would immediately lose its utility and selection would be impossible.

Biological systems also generate correlations. In particular, information transmission is an exercise in correlating input and output [3], and recent years have thus seen information theory applied to biological systems involved in sensing [4-6], signaling [7,8], chemotaxis [1,2], adaption [9,10], and beyond [11]. In the language of information theory, correlated variables $X$ and $Y$ have positive mutual information $I(X ; Y)=$ $\sum_{x \in X, y \in Y} p(x, y) \ln [p(x, y) / p(x) p(y)]$ (measured in nats), with $p(x, y)$ the joint probability of a given state and $p(x)$ and $p(y)$ the marginals. The "information entropy" $H(Y)=$ $-\sum_{y \in Y} p(y) \ln p(y)$ quantifies $Y$ 's uncertainty, and $I(X ; Y)$ is the reduction in this entropy given knowledge of $X$ : $I(X ; Y)=H(Y)-H(Y \mid X)$. The mutual information is symmetric, non-negative, and zero if and only if $X$ and $Y$ are statistically independent.

Information theory is also deeply connected to thermodynamics [12-20]. Sagawa and Ueda [14], building on Refs. [12,13], showed that measurement cycles have a minimal work cost equal to the mutual information generated between data and memory. Horowitz and Esposito showed that entropy production within a system $X$ can be negative if $X$ is coupled to a second system $Y$ and transitions in $X$ decrease $I(X ; Y)$ [18]. A third key result, essential to exorcising Maxwell's demon [6,21], is that if $X$ and $Y$ are uncoupled from each other, yet coupled to heat baths at temperature $T$, then the total free energy is [22,23]

$$
\tilde{F}(X, Y)=\tilde{F}(X)+\tilde{F}(Y)+k_{B} T I(X ; Y) .
$$

Here $\tilde{F}(X)=F_{\text {eq }}(X)-k T \sum_{x \in X} p(x) \ln \left[p_{\text {eq }}(x) / p(x)\right]$ is the nonequilibrium free energy [20,23], with the tilde indicating the generalization from the standard equilibrium free energy $F_{\text {eq }}(X)$. Systems $X$ and $Y$ could be two noninteracting spins or two physically separated molecules. For uncoupled systems, the partition function is separable, and $X$ and $Y$ are independent in equilibrium: $I_{\text {eq }}(X ; Y)=0$. However, correlations induced by coupling between $X$ and $Y$ at earlier times could persist even after the coupling has been removed [6], or $X$ and $Y$ may have been initialized by related processes, allowing $I(X ; Y)>0$. If $I(X ; Y)>0$ despite the current absence of interactions, $\tilde{F}(X, Y)>$ $F_{\text {eq }}(X, Y)=F_{\text {eq }}(X)+F_{\text {eq }}(Y)$ even if $\tilde{F}(X)=F_{\text {eq }}(X)$ and $\tilde{F}(Y)=F_{\text {eq }}(Y)$. Thus, $I(X ; Y)>0$ between uncoupled systems implies excess free energy, and excess free energy is a resource from which work can be extracted [20,23].

Information appears inherently abstract, and workperforming devices coupled to strings of zeros and ones [13,22,24-29] can seem remarkable. However, as Landauer pointed out, "information is physical" [30], and processing it calls for a physical realization. We propose biochemical information-exploiting devices to show both thermodynamically [via Eq. (1)] and physically (via the actual information-processing mechanism) how mutual information can be used to do work, setting the basis for more sophisticated information-exploiting devices.

Our first device is a "tape-driven" biochemical machine, which, unlike previous tape-driven devices [13,22,24-29], exploits mutual information within the input. The information-processing mechanism is explicit, differing from measurement-feedback devices previously considered $[15,22,31,32]$. A recent study did suggest work extraction from a perfectly correlated quantum tape [33]. However, like the majority of tape-driven machines [13,22, 24-26,28,29], the dynamics is not based on an actual physical system. It is also unclear how the initial state would be created or the mechanism generalized [33]. Using 
an explicit system emphasizes the constraints under which information-processing devices operate. For example, the consequences of externally controlling a tape that physically couples to the device are often ignored. With these effects in mind, we introduce a modified device that functions without a control, removing implicitly neglected costs, providing a simpler route to constructing an actual device, and moving closer to autonomous biological systems.

Our "engine" is an enzyme $E$, which is converted from inactive $(E)$ to active $\left(E^{\dagger}\right)$ by the binding of molecule $Y$. The enzyme can also bind to nucleotides ADP or ATP and a substrate $X$. When active, $E^{\dagger}$ catalyzes phosphate exchange between ATP and $X$ :

$X+\mathrm{ATP}+E^{\dagger} \rightleftharpoons E^{\dagger}-X$-ADP- $P_{i} \rightleftharpoons E^{\dagger}+X^{*}+\mathrm{ADP}$.

One natural enzyme-substrate-activator combination would be LBK1, AMPK and STRAD-MO25 [34], although others exist [35] and engineered examples [36,37] might be optimal. The active enzymatic engine transfers free energy from substrates (the fuel) to a bath of ATP or ADP (the load), or vice versa. The work done in converting a single ADP into ATP is given by the difference in chemical potentials, $\mu_{\mathrm{ATP}}-\mu_{\mathrm{ADP}}$. In dilute solutions, $\mu_{\mathrm{ATP}}-\mu_{\mathrm{ADP}}=$ $\mu_{\mathrm{ATP}}^{0}-\mu_{\mathrm{ADP}}^{0}+k_{B} T \ln ([\mathrm{ATP}] /[\mathrm{ADP}])$, with $\mu_{\mathrm{ATP}}^{0}-\mu_{\mathrm{ADP}}^{0}$ the "intrinsic" contribution. Changing $[\mathrm{ATP}] /[\mathrm{ADP}]$ thus adjusts the load.

As illustrated in Fig. 1, we pull a polymer decorated with substrates $X$ and $X^{*}$ past an enzyme tethered within a nucleotide bath. The regularly spaced substrates constitute "tape" $A$, with sites $A_{n}$. We shall discuss the second tape $B$, and operational details, later. The exploitable free energy per monomer stored in tape $A$ alone depends on the intrinsic chemical potentials of $X$ and $X^{*}, \mu_{X}^{0}$ and $\mu_{X^{*}}^{0}$, and the initial fraction of $X^{*}, q=p_{0}\left(A_{n}=X^{*}\right)$. Specifically, assuming $A_{n}$ are independent, $\tilde{F}\left(A_{n}\right)-F_{\text {eq }}\left(A_{n}\right)=$ $\left(q-q_{\mathrm{eq}}\right)\left(\mu_{X^{*}}^{0}-\mu_{X}^{0}\right)+k_{B} T\left[H_{\mathrm{eq}}\left(A_{n}\right)-H\left(A_{n}\right)\right]$, where $q_{\mathrm{eq}}$ is the equilibrium fraction of $X^{*}$ when the substrates are decoupled from ATP and ADP, $q_{\text {eq }}=p_{\text {eq }}\left(A_{n}=X^{*}\right)=$ $\left\{1+\exp \left[\left(\mu_{X^{*}}^{0}-\mu_{X}^{0}\right) / k_{B} T\right]\right\}^{-1}$. If left uncoupled from ATP and ADP, $A_{n}$ would eventually reach this equilibrium due to spontaneous transitions $X+P_{i} \leftrightarrow X^{*}$ that we assume are slow enough to be ignored under machine operation.

An active enzyme, supplied only with tape $A$, could do work on the bath if $\tilde{F}\left(A_{n}\right)-F_{\text {eq }}\left(A_{n}\right)>0$, implying an excess of the most unstable substrate, a low entropy $H\left(A_{n}\right)$, or both. If $\mu_{X}^{0}=\mu_{X^{*}}^{0}$ this $A$-only system is equivalent to the device of Ref. [24], with $X / X^{*}$ as degenerate zeros and ones storing free energy purely through $H\left(A_{n}\right)<$ $H_{\text {eq }}\left(A_{n}\right)=\ln 2$. Cao, Gong, and Quan [28] proposed using an enzyme in this context but without a physical tape. In these studies, it appears remarkable that abstract strings of (a)

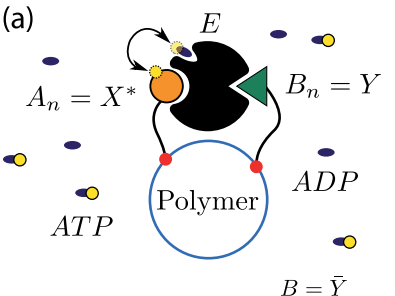

(c)
$A=X$

$A=X$
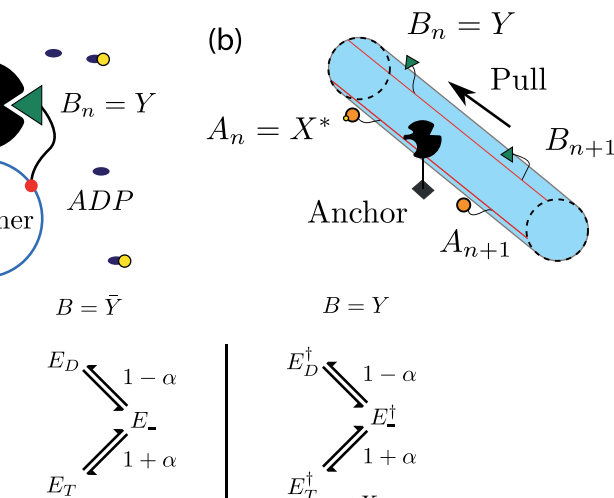

$B=Y$

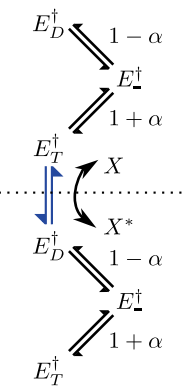

FIG. 1. (a) Schematic showing an enzyme interacting with the $n$th pair of sites, catalyzing $X^{*}+\mathrm{ADP} \rightleftharpoons X+\mathrm{ATP}$ on tape $A$ due to the presence of activator $Y$ on tape $B$. Tapes extend into and out of page, and yellow circles indicate phosphorylation. (b) Two tapes, coordinated on a single polymer, being pulled past a tethered enzyme. We propose a DNA origami-based construction in Ref. [38], Sec. 1. (c) State space of the model. $E_{T}$ and $E_{D}$ correspond to ATP- and ADP-bound enzymes, respectively, and $E_{-}$to a free enzyme. All unlabeled transitions have rate 1 . The blue arrows denote the reaction $X+\mathrm{ATP} \rightleftharpoons X^{*}+\mathrm{ADP}$.

bits can be used for work. In a physical implementation, however, the devices are powered by nonequilibrium fuel, like all machines. For general $\mu_{X}^{0}-\mu_{X^{*}}^{0}$, equilibrium states have $H_{\text {eq }}\left(A_{n}\right)<\ln 2$. One cannot, therefore, infer that tape $A$ is a fuel source from $H\left(A_{n}\right)$ alone; $\mu_{X}^{0}-\mu_{X^{*}}^{0}$ must be considered.

Our system has a second tape " $B$ " grafted onto the polymer, moving together with $A$ (Fig. 1). Each $n$ thus corresponds to a pair $A_{n}$ and $B_{n}$, with $B_{n}$ carrying $Y$ or an empty space $\bar{Y}$. The intersite interval is long enough that $E$ can interact with at most one pair simultaneously, and $A_{n}$ and $B_{n}$ are initially independent of $A_{m}$ and $B_{m}$ for $n \neq m$. Operational cycles begin with $E$ isolated from the sites on either tape and in equilibrium with respect to ATP or ADP binding. We then bring the next pair of sites $n$ alongside $E$. If $B_{n}=Y, E \rightarrow E^{\dagger}$ and conversion of $A_{n}$ between $X$ and $X^{*}$ according to Eq. (2) is possible ( $B_{n}$ remains unchanged). We then move the tapes along, allowing $E$ to relax back into equilibrium with the chemical bath. We return to the costs of external manipulation later. Tape $B$ provides an additional positive contribution to the exploitable free energy, $k_{B} T I\left(A_{n} ; B_{n}\right)$ [Eq. (1)]. Physically, the enzyme's decision-making interaction with $B_{n}$ sets its response to $A_{n}$, since $A_{n}$ is fixed when $B_{n}=\bar{Y}$. If $Y$ is correlated with $X^{*}$, then $X^{*}+$ ADP $\rightarrow X+$ ATP happens with increased frequency relative to $X+\mathrm{ATP} \rightarrow X^{*}+\mathrm{ADP}$, allowing work 
extraction even if $\tilde{F}\left(A_{n}\right)-F_{\text {eq }}\left(A_{n}\right)=0$. Unlike $H\left(A_{n}\right)<\ln 2$, $I\left(A_{n} ; B_{n}\right) \neq 0$ always implies stored free energy.

A proposal for instantiating the system, using a DNA origami assembly [39,40], is given in Sec. 1 of Ref. [38]. Here we analyze a simple model of device operation. Recall that $p_{0}\left(A_{n}=X^{*}\right)=q$. For simplicity, we introduce initial correlations through a single parameter $\psi$ :

$p_{0}\left(B_{n}=\bar{Y} \mid A_{n}=X^{*}\right)=p_{0}\left(B_{n}=Y \mid A_{n}=X\right)=\psi$,

$p_{0}\left(B_{n}=Y \mid A_{n}=X^{*}\right)=p_{0}\left(B_{n}=\bar{Y} \mid A_{n}=X\right)=1-\psi$.

We adjust the chemical load from the bath via $\alpha \in(-1,1)$ such that $[\mathrm{ATP}]=1+\alpha$ and $[\mathrm{ADP}]=1-\alpha$ relative to a reference concentration $C_{0}$.

We model tape manipulation as exposing $A_{n}$ to the enzyme for a time $\tau$, assuming that allostery and activator binding are sufficiently strong that the enzyme is active if $Y$ is present but inactive otherwise. Thus, the relevant transitions are binding or unbinding of nucleotides to $E$ and the catalysis of Eq. (2). Enzyme or substrate unbinding is assumed fast and catalysis instantaneous. For simplicity, we set all rate constants to unity $\left(1 / C_{0}\right.$ for bimolecular rate constants) and assume that $\mu_{\mathrm{ADP}}-\mu_{\mathrm{ATP}}=$ $k T \ln [(1-\alpha) /(1+\alpha)]$, implying that $\mu_{X}^{0}=\mu_{X^{*}}^{0}$ given these rate constants. We relax these assumptions in Ref. [38], Sec. 3. The model has the transition graph shown in Fig. 1(c), which specifies a reaction rate matrix $\mathcal{R}$ and probability density evolution $p_{t}=e^{\mathcal{R} t} p_{0}$. Since the ADP- or ATP-binding state of $E$ equilibrates between encounters with sites, and pairs of sites are initially independent, we can consider each pair $A_{n}, B_{n}$ separately. See Ref. [38], Sec. 2, for full dynamical solutions.

For $\tau \rightarrow \infty$, substrates paired with $B_{n}=Y$ relax to a new equilibrium with the bath. If $\alpha$ is positive, this equilibrium favors an excess of $X^{*}$, since $[\mathrm{ATP}]>[\mathrm{ADP}]$. The device can do work on the bath only if the substrates encountered by active enzymes are even more likely to be in state $X^{*}$ than this excess. For $q=0.5$ and $\mu_{X}^{0}=\mu_{X^{*}}^{0}$, tape $A$ is initially in equilibrium, $\tilde{F}_{0}\left(A_{n}\right)=F_{\text {eq }}\left(A_{n}\right)$, and thus cannot be used to do work without harnessing $I_{0}\left(A_{n} ; B_{n}\right)$. Correlations allow $E^{\dagger}$ to encounter an excess of $X^{*}(X)$ if $\psi<0.5(\psi>0.5)$, despite $q=0.5$. See Ref. [38], Sec. 2, for the resultant work and change in mutual information as a function of $\alpha$ and $\psi$.

We indicate operational regimes for $q=0.5$ in Fig. 2(a), using $\Delta$ to denote the change of a quantity during $\tau$. In the bottom right, $\Delta I\left(A_{n} ; B_{n}\right)<0$ and $\left\langle w_{\text {cycle }}\right\rangle>0$ : The correlations favor $X^{*}+$ ADP $\rightarrow X+$ ATP and are strong enough to do work against the load $\mu_{\text {ATP }}-\mu_{\text {ADP. }}$. This region is bounded by the loci $0_{W}$, along which $\left\langle w_{\text {cycle }}\right\rangle=0$ since the load is zero $\left(\mu_{\mathrm{ATP}}=\mu_{\mathrm{ADP}}\right)$, and $0_{\text {all }}(\alpha=1-2 \psi$, Ref. [38], Sec. 2), along which the correlations and load exactly balance, implying no net evolution and $\Delta I\left(A_{n} ; B_{n}\right)=\left\langle w_{\text {cycle }}\right\rangle / k_{B} T=0$. Directly above is a region with $\Delta I\left(A_{n} ; B_{n}\right)>0$ and $\left\langle w_{\text {cycle }}\right\rangle<0$, in which the chemical load is strong enough to do work on the tapes, creating information. Finally, above this regime, both $\Delta I\left(A_{n} ; B_{n}\right)<0$ and $\left\langle w_{\text {cycle }}\right\rangle<0$. These regimes are repeated on the lefthand side of the figure.

The final two regimes are divided by the locus $0_{I}$, along which $\Delta I\left(A_{n} ; B_{n}\right)=0$. The dynamics here is clarified by considering the covariance $\xi$ between $A_{n}$ and $B_{n}$, which quantifies not only the correlation strength but also whether $X^{*}$ is associated with $Y$ or $\bar{Y}$. Along $0_{I}, X^{*}$ typically appears with $\bar{Y}$ for $\alpha>0$ and with $Y$ for $\alpha<0$. Thus, both the load and correlations initially favor $X^{*} \rightarrow X$ for $\alpha<0$ and $X \rightarrow X^{*}$ for $\alpha>0$. As the reactions proceed, the correlations between $A_{n}$ and $B_{n}$ drop and eventually reverse as substrates are converted. The resultant excess of the alternative substrate opposes the chemical load and eventually halts the reaction at $\Delta I\left(A_{n} ; B_{n}\right)=0$ but with inverted correlations [Fig. 2(b)].
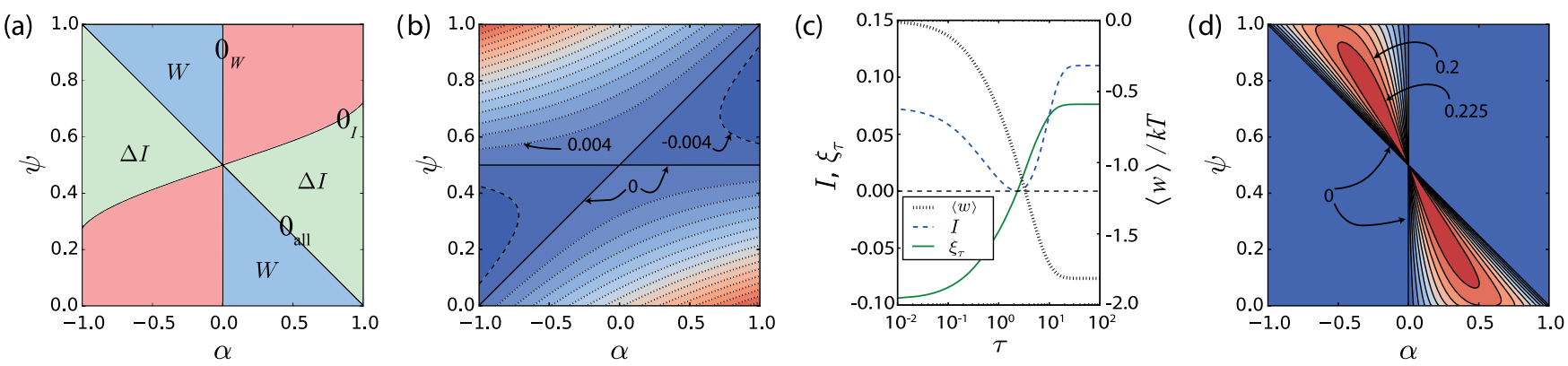

FIG. 2. (a) Regimes of operation for $q=0.5, \tau \rightarrow \infty$ as a function of chemical load $\alpha$ and correlation strength $\psi$ [see Eq. (3)]: $W$ indicates $\left\langle w_{\text {cycle }}\right\rangle>0$, and $\Delta I$ indicates $\Delta I\left(A_{n} ; B_{n}\right)>0 ;\left\langle w_{\text {cycle }}\right\rangle$ and $\Delta I\left(A_{n} ; B_{n}\right)$ are negative elsewhere. (b) Product of covariances $\xi_{0}\left(A_{n}, B_{n}\right) \xi_{\tau}\left(A_{n}, B_{n}\right)$ for $q=0.5, \tau \rightarrow \infty$, showing correlation reversal. Covariances calculated by taking $X^{*}$ and $Y$ as 1 and $X$ and $\bar{Y}$ as 0 . Negative contours are dashed lines, positive are dotted, and zero is solid (contours separated by units of 0.004). (c) Behavior of $\left\langle w_{\text {cycle }}\right\rangle, \Delta I\left(A_{n} ; B_{n}\right)$, and correlation $\xi_{\tau}\left(A_{n}, B_{n}\right)$ as a function of $\tau$ for $q=0.5, \alpha=0.99$, and $\psi=0.69$, showing nonmonotonicity of information. (d) Efficiency $\eta$ of converting the initial mutual information into work. Contours run downwards from 0.225 with a separation of 0.025 . 
Considering finite $\tau$ highlights the dynamics of correlation inversion; see Fig. 2(c) for $\alpha=0.99$ and $\psi=0.69$, parameters close to the $0_{I}$ locus at $\tau \rightarrow \infty$ (shifting the $0_{I}$ locus is the main effect of finite $\tau$; see Ref. [38], Sec. 3). The reversal of correlations with $\tau$ demonstrates the difference between each pair of sites $\left(A_{n}, B_{n}\right)$ and the chemical bath; they function like a charged capacitor and a constant voltage supply, respectively. If connected in series, a capacitor and supply can initially work together; eventually, however, the capacitor discharges and then recharges with the opposite polarity.

We plot the fraction of $I_{0}\left(A_{n}, B_{n}\right)$ converted into work, $\eta=\left\langle w_{\text {cycle }}\right\rangle / k_{B} T I_{0}\left(A_{n} ; B_{n}\right)$, in Fig. 2(d). The maximum $\eta$ is $\sim 1 / 4$, midway between $0_{\text {all }}$ and $0_{W}$. Near $0_{\text {all }}$, the system is near equilibrium, and the evolution involves negligible dissipation but also very little actual change during $\tau$, so $\Delta I\left(A_{n} ; B_{n}\right) \approx 0$. Near $0_{W}$, the system evolves significantly during $\tau$ but does so wastefully, as the chemical load is too small. See Ref. [38], Sec. 2, for a further analysis of the efficiency.

For $q \neq 0.5, \tilde{F}_{0}\left(A_{n}\right) \neq F_{\text {eq }}\left(A_{n}\right)$, and tape $A$ itself stores free energy. New regimes of behavior arise, shown in Fig. 3(a) for $q=0.7$. We see all sign permutations of $\Delta \tilde{F}\left(A_{n}\right), \Delta I\left(A_{n} ; B_{n}\right)$, and $\left\langle w_{\text {cycle }}\right\rangle$ except for the secondlaw-violating $\Delta \tilde{F}\left(A_{n}\right), \Delta I\left(A_{n} ; B_{n}\right),\left\langle w_{\text {cycle }}\right\rangle>0$. The initial value of $\tilde{F}\left(A_{n}\right) \neq F_{\text {eq }}\left(A_{n}\right)$ distorts regimes and introduces a new boundary $0_{F}$. Locus $0_{F}$ is analogous to $0_{I}$ : Along $0_{F}$, the probabilities of $X^{*}$ and $X$ are inverted by machine operation, preserving $\tilde{F}\left(A_{n}\right)=\tilde{F}_{0}\left(A_{n}\right)$.
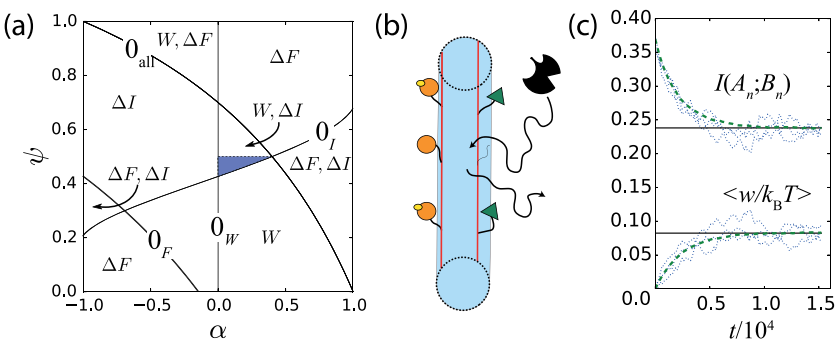

FIG. 3. (a) Regimes of behavior for $q=0.7, \tau \rightarrow \infty: \Delta F$ indicates $\Delta \tilde{F}\left(A_{n}\right)>0 ; W$ and $\Delta I$ are defined equivalently. The highlighted (blue) region has $\Delta I\left(A_{n} ; B_{n}\right) \geq 0$ and a greater $\left\langle w_{\text {cycle }}\right\rangle$ than for a system with the same $q, \alpha$, but $I_{0}\left(A_{n} ; B_{n}\right)=0$. (b) A completely autonomous device in which a freely diffusing enzyme interacts with correlated tapes. (c) Autonomous dynamics, with $q=0.5$, $\psi=0.1$, and $\alpha=0.5$. In addition to the rate constants in Fig. 1(c), it is necessary to define a rate at which the enzyme binds to activator $Y\left(k_{\text {bind }}=0.01\right.$ per activator here $)$ and an unbinding rate $\left(k_{\text {off }}=1\right.$ here). We plot the average work per site $\langle w\rangle / k T$ and $I\left(A_{n} ; B_{n}\right)$ obtained from 200 independent Gillespie simulations [41] of 1000 pairs of sites (dashed lines), showing that they converge to the values predicted in the $\tau \rightarrow \infty$ limit of the externally controlled device (solid lines). Dotted lines show information and work within three individual simulations (for subtleties in interpeting $I\left(A_{n} ; B_{n}\right)$ within a single trajectory, see Ref. [38], Sec. 6).
When tape $A$ itself is a nonequilibrium fuel, nonzero $I_{0}\left(A_{n} ; B_{n}\right)$ allows for increased $\left\langle w_{\text {cycle }}\right\rangle$ in two ways. The most intuitive is analogous to the $q=0.5$ case: $I\left(A_{n} ; B_{n}\right)$ is consumed to drive catalysis against the bath's chemical load. More surprisingly, in the highlighted region in Fig. 3(a), $\Delta I\left(A_{n} ; B_{n}\right) \geq 0$, yet $\left\langle w_{\text {cycle }}\right\rangle$ is increased relative to an otherwise equivalent system with $I_{0}\left(A_{n} ; B_{n}\right)=0$. Here, correlations initially support the drive from $\tilde{F}\left(A_{n}\right) \neq$ $F_{\text {eq }}\left(A_{n}\right)$ to do work against the bath but eventually reverse and oppose further reactions. The need to first reverse the initial correlations allows more ATP production before the reaction halts, enhancing $\left\langle w_{\text {cycle }}\right\rangle$ despite $\Delta I\left(A_{n} ; B_{n}\right) \geq 0$.

Each trajectory $z(t)=\left(e(t), a_{n}(t), b_{n}(t)\right)$, with $e(t)$, $a_{n}(t)$, and $b_{n}(t)$ representing the states of $E, A_{n}$, and $B_{n}$, respectively, over time, produces a stochastic entropy $\sigma\left[z(t), p_{t}(z)\right], \quad$ a functional of $z(t)$, and the probability of occupying state $z$ at time $t, p_{t}(z)$ [42-44]. With $b_{n}(t)$ constant, the fluctuation relation $\left\langle e^{-\bar{\sigma}\left[e_{n}(t), a_{n}(t), p_{t}\left(e, a_{n}\right)\right] / k_{B}+\Delta i_{0, \tau}\left(b_{n} ; e, a_{n}\right)}\right\rangle=1$ holds, where $\bar{\sigma}$ is the stochastic entropy generated by the $\left(E, A_{n}\right)$ subsystem and $i\left(b_{n} ; e, a_{n}\right)=\ln \left[p\left(b_{n}, e, a_{n}\right) / p\left(b_{n}\right) p\left(e, a_{n}\right)\right]$ is pointwise mutual information [17]. This result shows at the trajectory level how consumption of mutual information compensates for entropy reduction elsewhere, permitting $\Delta F\left(A_{n}\right)>0$ or $\left\langle w_{\text {cycle }}\right\rangle>0$. Unusually, in our model the fluctuation theorem is verifiable by explicit summation (Ref. [38], Sec. 4).

Figures 1(a) and 1(b) emphasize that the information register physically couples to the device. If the tapes are pulled at a finite rate, irreversible work will be done on the protein-protein interactions. Minimizing this dissipation through slow tape manipulation is problematic due to unwanted spontaneous transitions $X+P_{i} \leftrightarrow X^{*}$. Additional subtleties also arise when unidirectional motion is imposed externally [45], as outlined in Ref. [38], Sec. 5. These problems are eliminated if the device is autonomous, with no external manipulation after initiation. We therefore propose a modified autonomous system with an enzyme that diffuses freely between static sites [Fig. 3(b)]. The resultant dynamics are more complex-the enzyme can repeatedly return to the same sites - but $I_{0}\left(A_{n} ; B_{n}\right)$ still allows selective interaction with either $X$ or $X^{*}$. Indeed, autonomous systems in the long-time limit and nonautonomous systems with $\tau \rightarrow \infty$ perform identically. In both cases, the enzyme allows those substrates paired with $Y$ to relax to equilibrium with the bath, and thus the limiting distribution is the same for a given set of $\alpha, \psi$, and $q$. Thus, as shown in Fig. 3(c), the long-time average behavior of the autonomous system reproduces the previously reported behavior.

Many sophisticated behaviors exploit correlations in the environment, and we have taken the first step in designing minimal artificial systems that do this in the most fundamental way possible, by extracting work. Like biological systems, our second design can function autonomously. The chemical free energy stored could power a molecular 
motor, for example, and an eventual goal would be to design a minimal system that actually used the harvested free energy to support itself. Our devices lack memory, since the information exploited is the correlation between degrees of freedom that are encountered simultaneously. Designing systems that can harness correlations over time is the subject of future work.

T.E. O. acknowledges support from a Royal Society University Research Fellowship. The contributions of T. M. and N. S. J. were supported by BBSRC studentship $\mathrm{BB} / \mathrm{J} 014575 / 1$. This work is part of the research programme of the Foundation for Fundamental Research on Matter (FOM), which is part of the Netherlands Organisation for Scientific Research (NWO).

*t.ouldridge@imperial.ac.uk

[1] G. Micali and R. G. Endres, Curr. Opin. Microbiol. 30, 8 (2016).

[2] N. B. Becker, A. Mugler, and P. R. ten Wolde, Phys. Rev. Lett. 115, 258103 (2015).

[3] C. E. Shannon and W. Weaver, The Mathematical Theory of Communication (University of Illinois, Champaign, 1949).

[4] C. C. Govern and P. R. ten Wolde, Phys. Rev. Lett. 113, 258102 (2014).

[5] A. C. Barato, D. Hartich, and U. Seifert, New J. Phys. 16, 103024 (2014).

[6] T. E. Ouldridge, C. C. Govern, and P. R. ten Wolde, arXiv: 1503.00909.

[7] R. Cheong, A. Rhee, C. J. Wang, I. Nemenman, and A. Levchenko, Science 334, 354 (2011).

[8] W. de Ronde, F. Tostevin, and P. R. ten Wolde, Phys. Rev. Lett. 107, 048101 (2011).

[9] P. Sartori, L. Granger, C. F. Lee, and J. M. Horowitz, PLoS Comput. Biol. 10, e1003974 (2014).

[10] S. Ito and T. Sagawa, Nat. Commun. 6, 7498 (2015).

[11] W. Bialek, arXiv:1512.08954.

[12] R. Landauer, IBM J. Res. Dev. 5, 183 (1961).

[13] C. H. Bennett, Int. J. Theor. Phys. 21, 905 (1982).

[14] T. Sagawa and M. Ueda, Phys. Rev. Lett. 102, 250602 (2009).

[15] M. Bauer, D. Abreu, and U. Seifert, J. Phys. A 45, 162001 (2012).

[16] S. Still, D. A. Sivak, A. J. Bell, and G. E. Crooks, Phys. Rev. Lett. 109, 120604 (2012).

[17] T. Sagawa and M. Ueda, Phys. Rev. Lett. 109, 180602 (2012).
[18] J. M. Horowitz and M. Esposito, Phys. Rev. X 4, 031015 (2014).

[19] A. C. Barato and U. Seifert, Phys. Rev. Lett. 112, 090601 (2014).

[20] J. M. R. Parrondo, J. M. Horowitz, and T. Sagawa, Nat. Phys. 11, 131 (2015).

[21] P. N. Fahn, Found. Phys. 26, 71 (1996).

[22] J. M. Horowitz, T. Sagawa, and J. M. R. Parrondo, Phys. Rev. Lett. 111, 010602 (2013).

[23] M. Esposito and C. Van Den Broeck, Europhys. Lett. 95, 40004 (2011).

[24] D. Mandal and C. Jarzynski, Proc. Natl. Acad. Sci. U.S.A. 109, 11641 (2012).

[25] D. Mandal, H. T. Quan, and C. Jarzynski, Phys. Rev. Lett. 111, 030602 (2013).

[26] A. C. Barato and U. Seifert, Europhys. Lett. 101, 60001 (2013).

[27] Z. Lu, D. Mandal, and C. Jarzynski, Phys. Today 67, No. 8, 60 (2014).

[28] Y. Cao, Z. Gong, and H. T. Quan, Phys. Rev. E 91, 062117 (2015).

[29] A. B. Boyd, D. Mandal, and J. P. Crutchfield, New J. Phys. 18, 023049 (2016).

[30] R. Landauer, Phys. Today 44, No. 5, 23 (1991).

[31] S. Toyabe, T. Sagawa, M. Ueda, E. Muneyuki, and M. Sano, Nat. Phys. 6, 988 (2010).

[32] M. D. Vidrighin, O. Dahlsten, M. Barbieri, M. S. Kim, V. Vedral, and I. A. Walmsley, Phys. Rev. Lett. 116, 050401 (2016).

[33] A. Chapman and A. Miyake, Phys. Rev. E 92, 062125 (2015).

[34] E. Zeqiraj, B. M. Filippi, M. Deak, D. R. Alessi, and D. M. F. van Aalten, Science 326, 1707 (2009).

[35] S. W. Cowan-Jacob, W. Jahnke, and S. Knapp, Future Med. Chem. 6, 541 (2014).

[36] Y. Qiao, H. Molina, A. Pandey, J. Zhang, and P. A. Cole, Science 311, 1293 (2006).

[37] A. V. Karginov, F. Ding, P. Kota, N. V. Dokholyan, and K. M. Hahn, Nat. Biotechnol. 28, 743 (2010).

[38] See Supplemental Material at http://link.aps.org/ supplemental/10.1103/PhysRevLett.118.028101 for additional derivations and details of the design.

[39] P. W. K. Rothemund, Nature (London) 440, 297 (2006).

[40] S. M. Douglas, H. Dietz, T. Liedl, B. Högberg, F. Graf, and W. M. Shih, Nature (London) 459, 414 (2009).

[41] D. T. Gillespie, J. Phys. Chem. 81, 2340 (1977).

[42] G. E. Crooks, Phys. Rev. E 60, 2721 (1999).

[43] U. Seifert, Eur. Phys. J. E 34, 26 (2011).

[44] U. Seifert, Rep. Prog. Phys. 75, 126001 (2012).

[45] B. B. Machta, Phys. Rev. Lett. 115, 260603 (2015). 


\section{Publisher's Note: Biochemical Machines for the Interconversion of Mutual Information and Work [Phys. Rev. Lett. 118, 028101 (2017)]}

Thomas McGrath, Nick S. Jones, Pieter Rein ten Wolde, and Thomas E. Ouldridge (Received 13 January 2017; published 27 January 2017)

DOI: 10.1103/PhysRevLett.118.049902

This Letter was published online on 10 January 2017 with an omission of an affiliation. The author affiliations should read as

Thomas McGrath, ${ }^{1}$ Nick S. Jones, ${ }^{1}$ Pieter Rein ten Wolde, ${ }^{2}$ and Thomas E. Ouldridge ${ }^{3, *}$

${ }^{1}$ Department of Mathematics, Imperial College London, London SW7 2AZ, United Kingdom

${ }^{2}$ FOM Institute AMOLF, Science Park 104, 1098 XE Amsterdam, Netherlands

${ }^{3}$ Department of Bioengineering, Imperial College London, London SW7 2AZ, United Kingdom

The affiliations have been corrected as of 12 January 2017. The affiliations are correct in the printed version of the journal. 